Article

\title{
A New Approach to Calculate the Shielding Factor of Magnetic Shields Comprising Nonlinear Ferromagnetic Materials under Arbitrary Disturbances
}

\author{
Yiyang Zhao ${ }^{1}\left(\mathbb{D}\right.$, Zhiyin Sun ${ }^{1,2, *} \mathbb{D}$, Donghua Pan ${ }^{1,2}\left(\mathbb{D}\right.$, Shengxin Lin ${ }^{1}$, Yinxi Jin ${ }^{1,2}$ \\ and Liyi $\mathrm{Li}^{1,2, *}$ \\ 1 Department of Electrical Engineering, Harbin Institute of Technology, Harbin 150001, China; \\ zhaoyiyang0819@163.com (Y.Z.); pandonghua@hit.edu.cn (D.P.); 15546012112@163.com (S.L.); \\ jinyinxi2006@126.com (Y.J.) \\ 2 Laboratory for Space Environment and Physical Sciences, Harbin Institute of Technology, \\ Harbin 150001, China \\ * Correspondence: 23hnhosava@163.com (Z.S.); liliyi@hit.edu.cn (L.L.); Tel.: +86-0451-8640-2989 (Z.S.)
}

Received: 8 April 2019; Accepted: 24 May 2019; Published: 29 May 2019

\begin{abstract}
To enable the realization of ultra-low magnetic fields for scientific and technological research, magnetic shielding is required to create a space with low residual magnetic field and high shielding factors. The shielding factors of magnetic shields are due to nonlinear material properties, the geometry and structure of the shields, and the external magnetic fields. Magnetic shielding is used in environments full of random realistic disturbances, resulting in an arbitrary and random external magnetic field, and in this case, the shielding effect is hard to define simply by the shielding factors. A new method to simulate and predict a dynamic internal space magnetic field wave is proposed based on the Finite Element method (FEM) combined with the Jiles-Atherton (JA) model. By simulating the hysteresis behavior of the magnetic shields and establishing a dynamic model, the new method can simulate dynamic magnetic field changes inside magnetic shields as long as the external disturbances are known. The shielding factors under an AC external field with a sine wave and certain frequencies are calculated to validate the feasibility of the new method. A real-time wave of internal magnetic flux density under an AC triangular wave external field is simulated directly with the new method versus a method that splits the triangular wave into several sine waves by a Fourier transform, divides the shielding factors, and then adds the quotients together. Moreover, real-time internal waves under some arbitrary fields are measured. Experimental internal magnetic flux density waves of a 4-layer magnetically shielded room (MSR) at the Harbin Institute of Technology (HIT) fit the simulated results well, taking experimental errors into account.
\end{abstract}

Keywords: magnetic shields; shielding factor; Jiles-Atherton model; nonlinear ferromagnetic materials; environmental disturbances

\section{Introduction}

Magnetic shields with high shielding factors are required to create a space with magnetic noise at the $\mathrm{fT} / \sqrt{\mathrm{Hz}}$ level. These have crucial applications in the fields of weak magnetic field detection technology, aerospace, and precise next-generation experiments in fundamental research at low energies [1-3]. Shielding factors are the most common index to evaluate the shielding effect of magnetic shields under a DC external field or an AC external field with sine waves at certain frequencies.

The shielding factors of magnetic shields are due to nonlinear material properties, the geometry and structure of the shields, and the external magnetic fields. There are mutual methods to calculate 
the shielding factor. One method is using the analytical formulas; analytical formulas to calculate the shielding factors of a multilayer cube magnetic shield structure under AC external fields were derived in detail [4]. Another method is the Finite Element Method (FEM), which uses a surface instead of a domain to describe the thin shielding layer and defines the interface via the equations shown in Ref. [5]. A method to evaluate the effective permeability curve to be used in the FEM model has been proposed, increasing the accuracy of the simulation result of the shielding factor and makes it much closer to the experimental results [6]. Nonetheless, these methods still have some limitations listed as follows:

First, traditionally, a material's magnetic property is represented by a relative permeability curve varying with magnetic field. Magnetic shields are usually made of permalloy, a high-permeability material. In the calculation with the analytical formulas, the relative permeability is seen as a constant turning the nonlinear material property to a linear one. In addition, the shielding material of an inner layer has lower magnetic field. It is difficult to find a suitable permeability constant for each layer. Although the relative permeability curve can be analyzed as a variable value by importing the basic magnetization curve using the FEM method, it still neglects the hysteresis characteristic of the shielding material, which is a key property of soft magnetic materials.

Second, the analytical formula can only theoretically calculate the shielding factor precisely for a few typical geometries, such as spherical shields and infinite cylindrical shields in the transverse direction, while the formulas for commonly used geometry shields, such as cubic magnetically shield rooms (MSRs) and finite cylindrical shields, are approximated by an infinite cylindrical shield. Thus, the approach is not precise.

Third, traditional methods can only give a numerical value of the shielding factor when the external magnetic fields are static or alternating but cannot provide the magnetic properties of a single point in the shielding layer or simulate a real-time curve of the internal magnetic field or magnetic flux density. When the external magnetic field is periodic, a method to calculate the internal magnitude of the magnetic field is proposed: split the external wave into several sine waves by a Fourier transform, divide the shielding factors at each frequency and add the quotients together. The external magnetic field might be disturbed by the environment in random and arbitrary ways at different times. When the external field is non-periodic, considering the complicated magnetic properties of the shielding material, it is difficult to analyze the shielding factors simply by taking a Fourier transform of the irregular field.

It is necessary to establish a dynamic model to analyze the dynamic internal magnetic field to improve the accuracy of the calculation of the shielding factors of different kinds of structures and geometries under different kinds of arbitrary external magnetic fields. This paper proposes a new method combining the Jiles-Atherton (JA) model to simulate the hysteresis behaviors of magnetic shields under arbitrary external fields. The JA model describes the hysteretic behavior in detail based on physical meanings, which can analyze energy changes in the magnetic domains during magnetization. This provides an accurate shielding factor analysis in the represence of random disturbances.

\section{Analytical Model}

The shielding factor of an MSR in this paper is defined as:

$$
S=\frac{B_{e x}}{B_{\text {in }}}
$$

where $B_{e x}$ is the magnitude of the magnetic flux density without shielding, and $B_{i n}$ is the magnitude of the internal magnetic flux density with shielding at the same point. In this paper, the magnetic flux density of the center point of the MSR is used to calculate the shielding factor. 


\subsection{Analytical Formula}

At present, the best MSRs in the world have a multilayer cubic shape, such as BMSR-2 in Physikalisch-Technische Bundesanstalt (PTB) [7] in Berlin and the MSR with an insert at Technische Universität München (TUM) [8]. The analytical formulas for the shielding factor of a 3-layer cubic MSR is [6]:

$$
\begin{gathered}
S=1+0.8 \frac{\mu_{r} d}{a} \\
S=1+S_{1}+S_{2}+S_{3}+0.8 S_{1} S_{2} S_{3}\left(1-\frac{a_{1}^{3}}{a_{2}^{3}}\right)\left(1-\frac{a_{2}^{3}}{a_{3}^{3}}\right)
\end{gathered}
$$

where $\mu_{r}$ is the relative permeability of the shielding material, $d$ is the thickness of the shield, and $a$ is the base length of the cubic.

\subsection{The Commonly Used FEM Method}

There are mutual FEM methods that use a surface instead of a domain to describe the shielding layer because there is a large difference in the order of magnitude of the dimensions between the base length of a cube, the air gap, and the thickness of the magnetic shield, which leads to difficulties in meshing the structure of the MSR [5]. The transition boundary condition in commercial software COMSOL is used in this paper. The interface for AC magnetic field is defined as Equations (4)-(7) [5]. It equates the shielding layer into a geometric thin layer, describing the discontinuity of the tangential electric field on both sides of the thin layer, through which the magnetic fields on both sides of the thin layer can be calculated.

$$
\begin{gathered}
\vec{n} \times \vec{H}_{1}=\vec{J}_{s 1} \\
\vec{n} \times \vec{H}_{2}=\vec{J}_{s 2} \\
\vec{J}_{s 1}=\frac{Z_{s} \vec{E}_{t 1}-Z_{t} \vec{E}_{t 2}}{Z_{s}^{2}-Z_{t}^{2}} \\
\vec{J}_{s 2}=\frac{Z_{s} \vec{E}_{t 2}-Z_{t} \vec{E}_{t 1}}{Z_{s}^{2}-Z_{t}^{2}}
\end{gathered}
$$

where $\vec{n}$ represents the normal direction of the shielding layer, $\vec{H}_{1}$ and $\vec{H}_{2}, \vec{E}_{t 2}$ and $\vec{E}_{t 2}$ refer to the magnetic field and the electric field of the different sides of the layer. $Z_{s}$ is the surface wave impedance, $Z_{t}$ is the transmission impedance of the shielding layer, and they are defined as Equations (8)-(10):

$$
\begin{gathered}
Z_{s}=\frac{-j \omega \mu}{k_{1}} \frac{1}{\tan \left(k_{1} d\right)} \\
Z_{t}=\frac{-j \omega \mu}{k_{1}} \frac{1}{\sin \left(k_{1} d\right)} \\
k_{1}=\omega \sqrt{(\epsilon+(\sigma /(j \omega))) \mu}
\end{gathered}
$$

The transition boundary condition section has these following material properties for the thin layer, which this boundary condition approximates: relative permittivity $\epsilon$, relative permeability $\mu$, electrical conductivity $\sigma$ and surface thickness $d$. In the equations, $j$ represents the imaginary unit, $k_{1}$ represents the wave number. Different values or expressions can be entered to define the shielding material. The shielding factor can be simulated by importing the permeability curve which can be found in material handbooks, while inputting the other parameters as constants. 


\subsection{The FEM Method Combined with the JA Model}

Traditional FEM methods used to calculate the shielding factor leads only to numerical results because the hysteretic behavior of the high permeability of the shielding material is neglected and is replaced by the relative permeability. To describe the hysteretic behavior, a new FEM method is proposed: combine the shielding material domain with the JA model, which is based on the analysis of the energy changes in the magnetic domains during magnetization $[9,10]$. In JA model, we use anhysteretic magnetization $M_{a n}$ to describe the ideal state of magnetization. $M_{a n}$ is depended on the effective field $H_{e}$, and is approximated by a Langevin function. The ideal energy of the magnetization $M$ is equal to the magnetostatic energy plus the dissipated loss due to pinning sites. Through the energy equation, the magnetization $M$ could be calculated.

To describe the magnetization process in any direction of shielding materials, the JA model can be described by a set of vector equations [11]. While demagnetizing, the dynamic magnetization process of the shielding material can be obtained by solving the vectorial equations of the JA model written in the partial differential equations (PDEs) interface in COMSOL [12]. In this paper, we use the material model Jiles-Antherton Isotropic material to describe the JA model instead of the PDEs interface in COMSOL. Similarly, the dynamic properties of the shielding material during the magnetic shielding process can be obtained. Meanwhile, the internal dynamic magnetic field curve can also be simulated by the method. After building an arbitrary geometry in FEM software COMSOL 5.3, the domain of permalloy is defined by selecting its material as JA anisotropic hysteretic material and the equations are as follows:

$$
\begin{gathered}
\frac{d \vec{M}}{d t}=\left[\frac{1}{k}\left(\vec{M}_{a n}-\vec{M}\right) \cdot \frac{d \vec{H}_{e}}{d t}\right] \frac{\frac{1}{k}\left(\vec{M}_{a n}-\vec{M}\right)}{\left|\frac{1}{k}\left(\vec{M}_{a n}-\vec{M}\right)\right|}+c \frac{d \vec{M}_{a n}}{d t} \\
\vec{H}_{e}=\vec{H}+\alpha \vec{M} \\
\vec{M}_{a n}=\vec{M}_{s}\left(\operatorname{coth}\left|\frac{\vec{H}_{e}}{a}\right|-\left|\frac{a}{\vec{H}_{e}}\right|\right) \frac{\vec{H}_{e}}{\left|H_{e}\right|}
\end{gathered}
$$

where $M_{s}$ is the saturated magnetization, $a$ is the domain wall density, $\alpha$ is a mean field parameter representing the coupling of domains, $k$ is the average energy required to break a pinning site, $c$ is the coefficient of the reversible domain wall bending during field changes.

The parameters including the conductibility of the aluminum and the permalloy can be defined by users.

\section{Experiment}

On one hand, the aim of this experiment is to measure the shielding factors of the MSR at different frequencies to verify the accuracy of the new FEM method combining the JA model to calculate shielding factors. On the other hand, the real-time curve of the magnetic flux density inside the MSR can be observed under the arbitrary external magnetic field waves that are generated by a coil system.

The MSR at the Harbin Institute of Technology (HIT) shown in Figure 1 is a cubic magnetic shield consisting of three layers of permalloy and a layer of aluminum, while the aluminum layer is fixed to the outermost permalloy layer through wooden structures. Each layer of the shield walls of the MSR in HIT is connected together actually, the unconnected walls you can see from the picture are some boards to protect the exciting coils physically from other things in the workshop where the MSR is placed and they're not a part of the shielding walls. The dimensions of each layer are shown in Table 1. The width of the permalloy plate is $350 \mathrm{~mm}$, which is limited by the heat treatment furnace. The layer of the MSR is vertically and horizontally lapped with narrow lap strips. 
Table 1. Dimensions of each layer of the MSR.

\begin{tabular}{ccccc}
\hline Layer & Material & Thickness & Side Length & Door Size \\
\hline 1 & Permalloy & $2 \mathrm{~mm}$ & $2.6 \mathrm{~m}$ & $2.2 \mathrm{~m} \times 2.5 \mathrm{~m}$ \\
2 & Aluminum & $8 \mathrm{~mm}$ & $2.43 \mathrm{~m}$ & $/$ \\
3 & Permalloy & $2 \mathrm{~mm}$ & $2.25 \mathrm{~m}$ & $0.8 \mathrm{~m} \times 1.80 \mathrm{~m}$ \\
4 & Permalloy & $2 \mathrm{~mm}$ & $1.85 \mathrm{~m}$ & $0.8 \mathrm{~m} \times 1.76 \mathrm{~m}$ \\
\hline
\end{tabular}

The center magnetic flux density of the MSR is measured by a three-axis magnetic flux gate Mag-03 manufactured by Bartington Company. The internal noise is $>10$ to $20 \mathrm{pTrms} / \sqrt{\mathrm{Hz}}$ at $1 \mathrm{~Hz}$, the orthogonality error is $<0.1^{\circ}$, and the alignment error is $<0.1^{\circ}$.

The external magnetic fields are generated by the 3-axis square Helmholtz coil shown in Figure 1. The magnetic flux density in the central point of the coil system before the MSR is placed can be calculated by Equation (14):

$$
B_{0}=\frac{4 \mu_{0} N I l^{2}}{\pi\left(l^{2}+a^{2}\right) \sqrt{2 l^{2}+a^{2}}}
$$

where $N$ represents the number of turns of the coils on a single coil frame, $I$ represents the current given to one turn of coil, $l$ represents half the length of the coil, and $a$ represents half the distance between the two identical square coils placed symmetrically along a common axis. For the coils implemented around the MSR, $l$ is $1.88 \mathrm{~m}, N$ is 10 and $a$ is $1.05 \mathrm{~m}$.

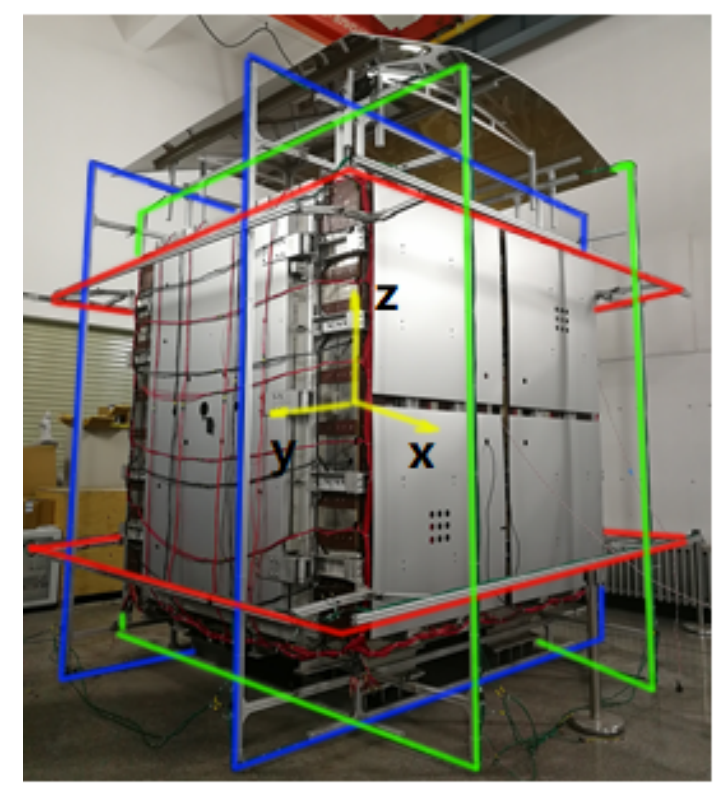

Figure 1. The MSR and the square Helmholtz coils at HIT.

\section{The Simulation Model of the JA Model Method}

A 2D model is built in COMSOL as shown in Figure 2a to verify the new FEM method combined with the JA model. The model contains infinite element domains in the outer layer cylindrical area and finite element domains, including the JA anisotropic hysteretic material domains, the aluminum domains, and the air domains. The geometry meshing is shown in Figure $2 \mathrm{~b}$. The meshing types of the permalloy and the aluminum layers are mapped, and the mesh type of the air domains is free triangular. The model is divided into 36,222 domain elements and 3508 edge elements and the number of degrees of freedom of the solution is 118,237 . 


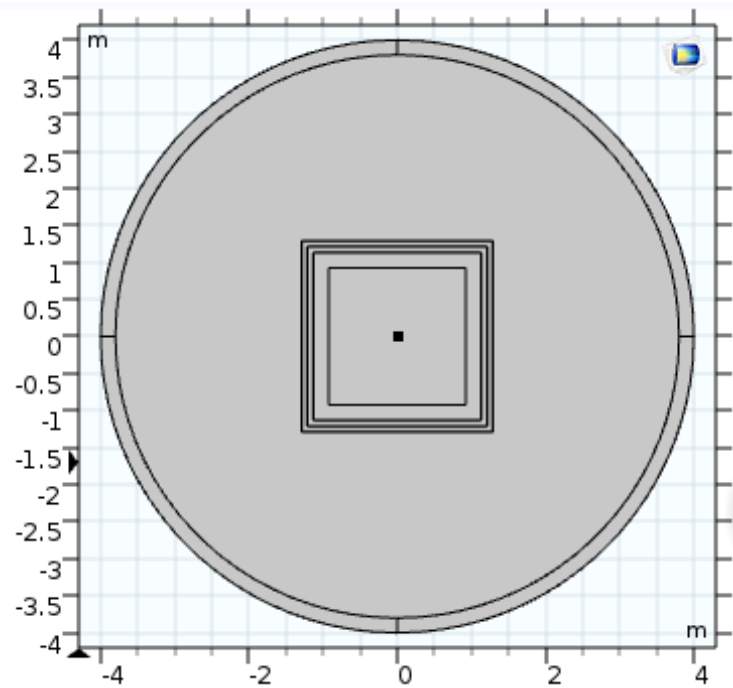

(a)

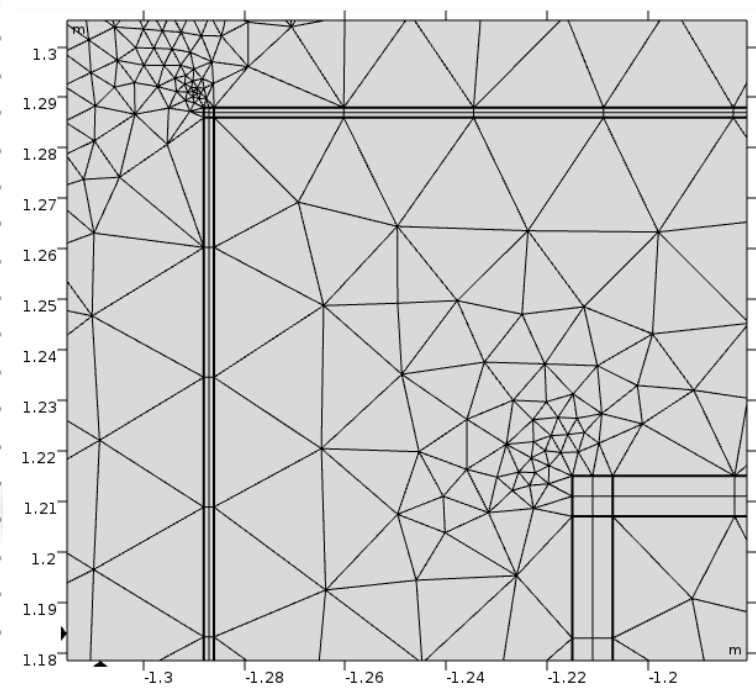

(b)

Figure 2. Geometry and meshing of the model. (a) The 2D geometry model of the MSR at HIT. (b) The meshing details of the model.

By selecting an option to solve for reduced field and then selecting the background field specification as uniform magnetic flux density, the expressions of the components of the external magnetic flux density can be entered. In this model, the y component is set to be zero while the expression of the $\mathrm{x}$ component is a function of time. By the way, the dimensions of each layer is set as shown in Table 1 and the parameters of the JA anisotropic hysteretic material is set as shown in Table 2 [13]. It has to be mentioned that the hysteresis characteristics of the shielding walls can be tested by permalloy ring samples. Due to the actual processing technology of ring samples and other factors, there will be some deviation between the measured hysteresis loop and the actually processed hysteresis loop of the shielding wall. Therefore, the JA model parameters of permalloy from Jiels are used in this paper [13].

Table 2. Main parameters of the JA anisotropic hysteretic material.

\begin{tabular}{cc}
\hline Items & Material \\
\hline $\mathrm{Ms}$ & $8 \times 10^{5} \mathrm{~A} / \mathrm{m}$ \\
$\mathrm{a}$ & $3.75 \mathrm{~A} / \mathrm{m}$ \\
$\alpha$ & $1.5 \times 10^{-5}$ \\
$\mathrm{k}$ & $2.4 \mathrm{~A} / \mathrm{m}$ \\
$\mathrm{c}$ & 0.35 \\
$\sigma$ & $1.8 \times 10^{6} \mathrm{~S} / \mathrm{m}$ \\
\hline
\end{tabular}

\section{Results}

\subsection{The Shielding Factors When the External Fields Are Sine Waves}

The shielding factors of the 2D MSR model above are calculated in different external sine magnetic flux densities to test the accuracy of the methods, including the traditional FEM method and the JA model method. Figure 3 shows that both the simulation results of the two methods are several times higher than the experiment result at higher frequencies with the greatest difference being 10 times at $1 \mathrm{~Hz}$. However, the simulation result is closer to the experimental results at lower frequencies, expecially at $0.01 \mathrm{~Hz}$, as shown in Figure 4; the normalized root-mean-square deviation (NRMSD) of the traditional FEM method is 0.5410 and for the JA method it is 0.3091 . 


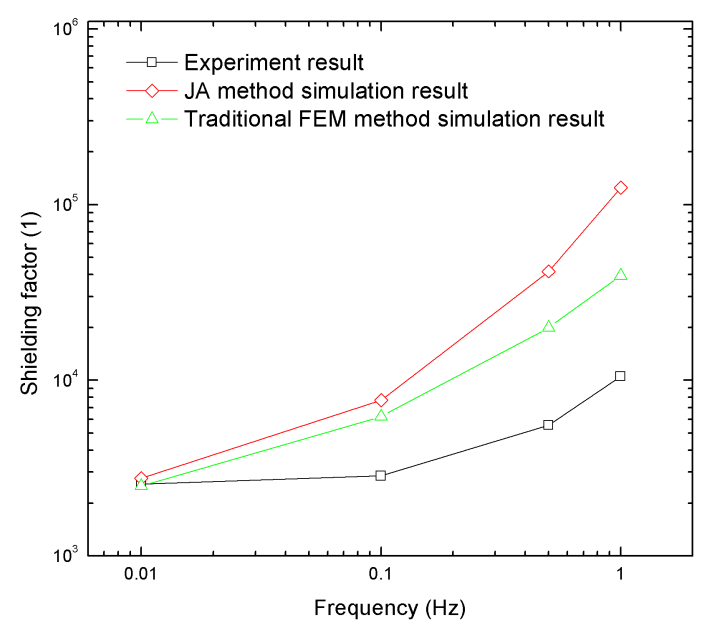

Figure 3. The shielding factors of the MSR at HIT when the magnitude of the external field is $50 \mu \mathrm{T}$.

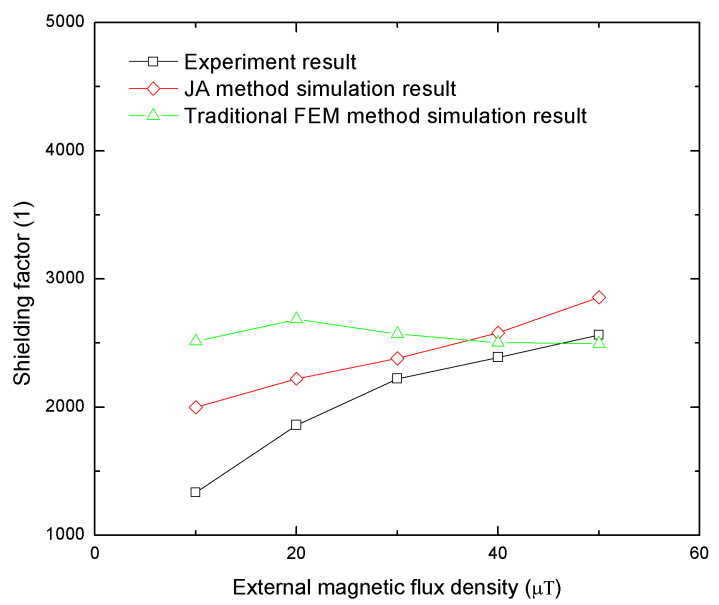

Figure 4. The shielding factors of the MSR at HIT when the frequency of the external field is $0.01 \mathrm{~Hz}$.

There are some reasons for the errors between the simulation and the experiment results. (1) The actual MSR is made of pieces of a permalloy plate, and the air gap between these plates can cause magnetic flux leakage, which reduces the shielding factor of the MSR [14]. In addition, the actual MSR has a door in each layer, and several holes are distributed in each surface, edge, and corner, which will influence the shielding factor. (2) The aluminum layer has reeds to make sure the door can be contacted more closely and it has some holes, too; as a result, the equivalent conductivity of the aluminum layer is reduced.

\subsection{The Internal Wave When the External Field Is a Triangle Wave}

When calculating the AC shielding factor, the traditional method usually considers the waveform of the external magnetic field to be sinusoidal. When the external magnetic field is non-sinusoidal, like triangular wave, the new shielding factor is worth discussing using both traditional method and the JA method to test the effect of the new method. As can be seen from Figure 3, the accuracy of the new method is better when the frequency of the external magnetic field is $0.01 \mathrm{~Hz}$. In order to study the shielding factor of triangular waves, we take a triangular external magnetic flux density wave with a frequency of $0.01 \mathrm{~Hz}$ and an amplitude of $50 \mu \mathrm{T}$ as an example and calculate its shielding factor by different methods including the JA model method and Fourier decomposition method described bellow: 
We can split the periodic triangular wave into several sine waves by using the Fourier transform as Equations (15) and (16), neglect the harmonic components with frequencies higher than $0.07 \mathrm{~Hz}$, divide their own shielding factors, and add them together to reform the internal triangular wave.

$$
\begin{gathered}
B_{e x}(t)=B_{1}\left\{\sin (2 \pi f t)-\frac{1}{3^{2}} \sin [2 \pi(3 f) t]+\frac{1}{5^{2}} \sin [2 \pi(5 f) t]-\frac{1}{7^{2}} \sin [2 \pi(7 f) t]+\cdots\right\} \\
B_{1}=\frac{8 A}{\pi^{2}}
\end{gathered}
$$

where $A$ and $f$ are the amplitude and the frequency of the external magnetic flux density. The sinusoidal component of each frequency has a shielding factor according to the simulated results shown in Figure 4. Thus, the internal magnetic flux density is:

$$
B_{\text {in }}(t)=\left\{\frac{1}{S_{1}} \sin (2 \pi f t)-\frac{1}{3^{2}} \frac{1}{S_{3}} \sin [2 \pi(3 f) t]+\frac{1}{5^{2}} \frac{1}{S_{5}} \sin [2 \pi(5 f) t]-\frac{1}{7^{2}} \frac{1}{7_{3}} \sin [2 \pi(7 f) t]+\cdots\right\}
$$

Figure 5 shows that the wave calculated by the Fourier decomposition method has the same magnitude as that calculated by the new method, but it can be seen that the Fourier decomposition method curve cannot show the hysteresis property, unlike the JA method. In the first period, the black curve does not look like the following curve. This is because in the simulation model, the initial magnetization of the shielding material domain is set to zero. After one period, the initial state of each period becomes the same. This is the reason why there is a phase difference between the result of the JA model method and the experimental results.

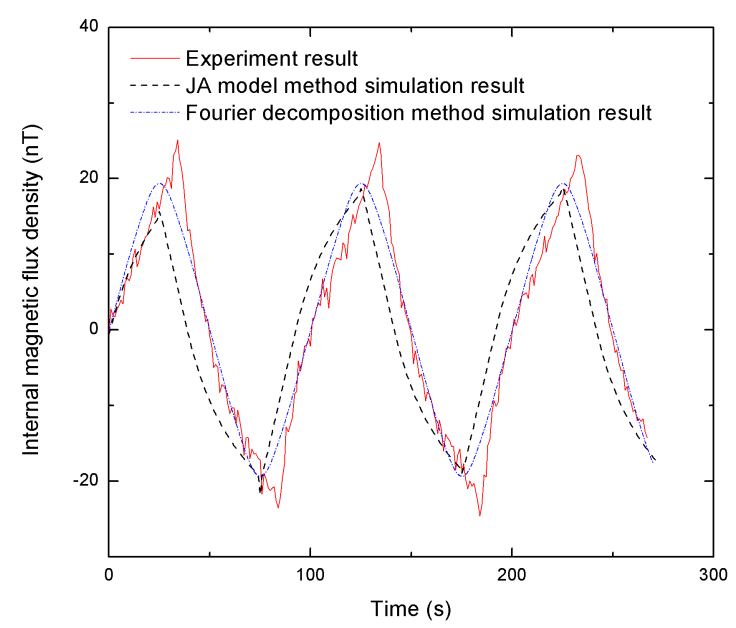

Figure 5. The internal wave of the MSR at HIT when the external field is a $0.01 \mathrm{~Hz}, 50 \mu \mathrm{T}$ triangular wave.

\subsection{The Internal Waves When the External Fields Are Arbitrary}

To verify the feasibility of the new method to simulate the internal dynamic wave of the magnetic flux density, we consider several non-periodic arbitrary external magnetic flux density waves containing of half triangular waves, half sine waves, slope waves and DC waves. There are 2 axes in Figures 6 and 7, showing the external and internal magnetic flux densities in one figure.

In Figure 6, the maximum external magnetic flux density is $50 \mu \mathrm{T}$. For the half triangle and the half sine wave, the amplitudes of each is $30 \mu \mathrm{T}$ and $40 \mu \mathrm{T}$, respectively, and the offset is $10 \mu \mathrm{T}$. Thus, the maximum of the internal wave is hard to calculate by the analytical formula or the traditional FEM method. The experiment curve shows that for half waves with a DC offset, the internal wave is not symmetrical about its axis of symetry. The offset is different before the half triangle and after the half sine wave. The simulation curve using the JA method agrees with the charicteristics above. The numerical value of the internal wave has a maximum point that basiclly agrees with the 
experimental result; however, there are some differences in the offset because there are DC offsets in the external field and the simulation did not consider this.

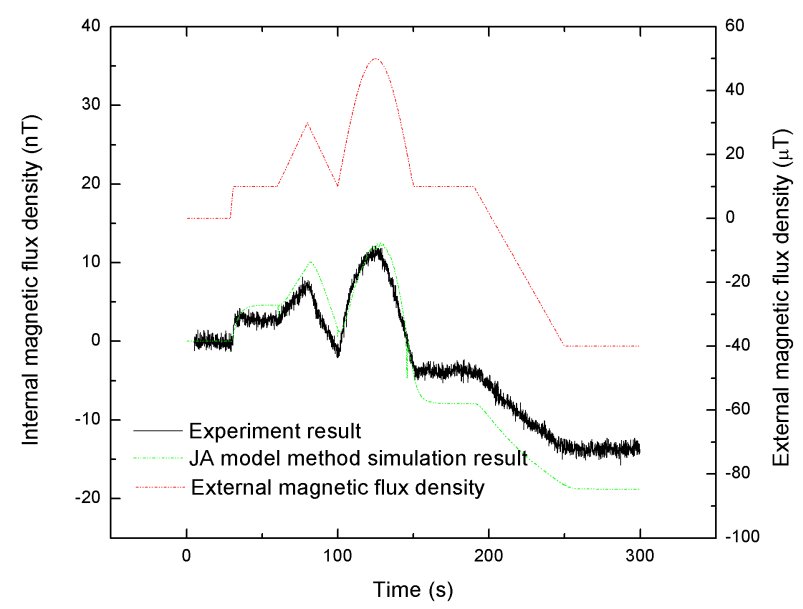

Figure 6. The wave of the MSR at HIT when the external field is arbitrary.

Figure 7 shows a situation in which the external wave contains a pulse. The pulse width is $0.5 \mathrm{~s}$, and the sampling time of the magnetic flux gate is $0.1 \mathrm{~s}$. The step size of the simulation model is set to be $0.2 \mathrm{~s}$, but it is changing during the solution. When the waveform has a sudden change like a step or a pulse, the time step will be decreased to make the solution more precise. The reciprocal of step size of the time-dependent solver of this model is varied from 5 to $1000 \mathrm{~s}^{-1}$ for different rates of field changes. The basic shape of the internal waveform simulated by the JA model method fits the experimental result. The differences between the simulation and the experiment is reasonable because the simulation did not take the complexity of the MSR structure, e.g., holes, air gaps and doors, which has influences on the real shielding performance.

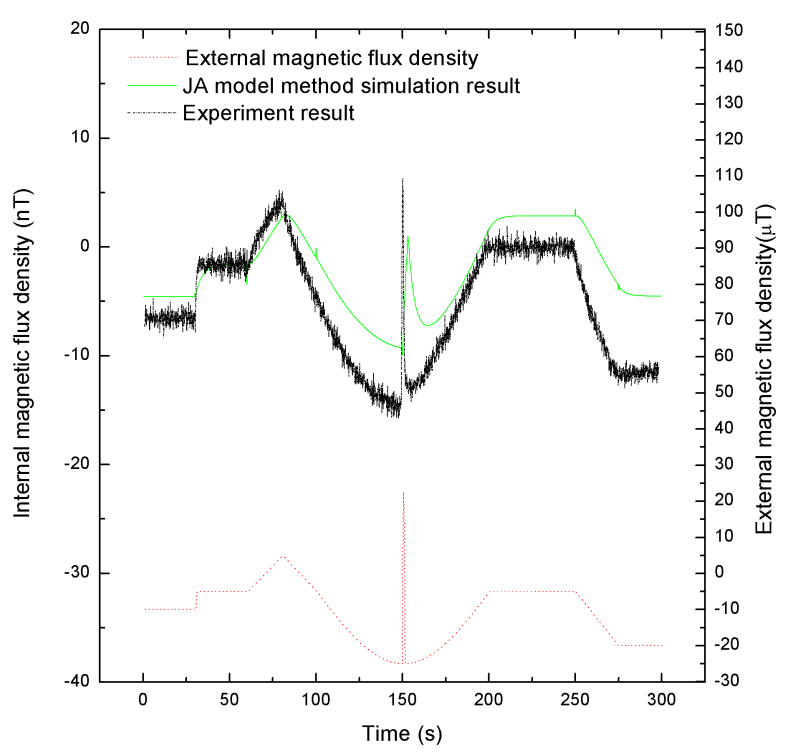

Figure 7. The wave of the MSR at HIT when the external field is arbitrary with a pulse.

\section{Discussion}

A new FEM method combined with the JA model is established to simulate the dynamic internal magnetic field of an MSR for various kinds of external magnetic fields. Three kinds of external magnetic flux density waveforms are simulated using the proposed JA model method. Experimental 
and simulation results of traditional FEM methods are compared to the simulated time domain curves by the JA model method. The basic consistence between these results proves the feasibility of the new method when calculating shielding factors for low frequencies. More important thing is that the new method has a distinct advantage over the traditional FEM methods and the Fourier decomposition method. On one hand, the JA method can simulate the dynamic internal wave since the shielding material has hysteresis characteristics, which improves the simulation precision of ultra-low magnetic field compare to normal shielding factor calculation method just using the permeability of the material. On the other hand, it can simulate the performance of magnetically shields under not only periodical external magnetic fields waves such as sine waves and triangle waves, but also arbitrary disturbances without periodicity which cannot easily be Fourier decomposed. Overall, this is the first time one can calculate the arbitrary external field influences on magnetically shields considering a material's hysteresis characteristics.

It needs to be mentioned that the simulations done in this paper uses DC JA model and also does not take into account the air gaps in the geometrical structure of the real MSR, resulting in higher shielding factor predictions. The advantage of this calculation method is that it can be extended to high-frequency calculation by using the frequency dependent JA model and the equivalent conductivity. As the common limitations of FEM method, it takes time or even impossible for complicated models which contains thin sheets, which is exactly the difficulties induced by layer structures and the air gaps in MSRs. If more accurate performance prediction for higher frequencies-especially around resonance frequency of the room is pursued, it is necessary to extend this method to 3D model with more complex structural characteristics, as long as one finds the solution of meshing or finds the proper equivalent parameters of the JA model and conductivity of the shielding wall. By the way, another source of the errors between the simulation results and the experiment results is the production errors of shielding material. The production process of shielding materials should be strictly controlled if the shielding factor error between designed value and actual value is to be reduced. In practice, the method can be used to make predictions as long as researchers know the disturbances outside of the MSR. The purpose of using coils to generate different kinds of external fields in this paper is to make accurate comparison analysis between experimental and theoretical results. It is believed that this method will provide the manufacturers and users of MSRs with much more realistic predictions and analysis, also a help to understand the various field fluctuation observed in real experiments, e.g., MEG measurement and calibration procedures, etc.

Author Contributions: Conceptualization, Y.Z. and Z.S.; methodology, Y.Z. and Z.S.; software, Z.S.; validation, D.P., Z.S., and Y.Z.; formal analysis, Z.S.; investigation, S.L.; resources, L.L.; data curation, Y.Z.; writing-original draft preparation, Y.Z.; writing-review and editing, Z.S.; visualization, Y.Z. and Y.J.; supervision, D.P.; project administration, D.P.; funding acquisition, L.L.

Funding: This research was supported by the Funds for International Cooperation and Exchange of the National Natural Science Foundation of China, grant number 51861135308, and the Young Scientists Fund of the National Natural Science Foundation of China, grant number 51807039 and the International Postdoctoral Exchange Fellowship Program, grant number No. 20171023.

Acknowledgments: We thank LetPub (www.letpub.com) for its linguistic assistance during the preparation of this manuscript.

Conflicts of Interest: The authors declare no conflict of interest.

\section{References}

1. Fratter, I.; Léger, J.-M.; Bertrand, F.; Jager, T.; Hulot, G.; Brocco, L.; Vigneron, P. Swarm Absolute Scalar Magnetometers first in-orbit results. Acta Astronaut. 2016, 121, 76-87. [CrossRef]

2. Matsushima, M.; Tsunakawa, H.; Iijima, Y.-I.; Nakazawa, S.; Matsuoka, A.; Ikegami, S.; Ishikawa, T.; Shibuya, H.; Shimizu, H.; Takahashi, F. Magnetic Cleanliness Program Under Control of Electromagnetic Compatibility for the SELENE (Kaguya) Spacecraft. Space Sci. Rev. 2010, 154, 253-264. [CrossRef] 
3. Heil, W.; Gemmel, C.; Karpuk, S.; Sobolev, Y.; Tullney, K.; Allmendinger, F.; Schmidt, U.; Burghoff, M.; Kilian, W.; Knappe-Grüneberg, S. Spin clocks: Probing fundamental symmetries in nature. Ann. Der Phys. 2013, 525, 539-549. [CrossRef]

4. Kelha, V.; Pukki, J.; Peltonen, R.; Penttinen, A.; Ilmoniemi, R.; Heino, J. Design, construction, and performance of a large-volume magnetic shield. IEEE Trans. Magn. 1982, 18, 260-270. [CrossRef]

5. Sun, Z.; Li, L.; Pan, D.; Liu, T.; Peter, F. Calculation Method of the Shielding Factor for Large-Scale Zero Magnetic Field Facility. Trans. China Electrotech. Soc. 2018, 33, 4450-4457. [CrossRef]

6. Liyi, L.; Zhiyin, S.; Donghua, P.; Jiaxi, L.; Feng, Y. An Approach to Analyzing Magnetically Shielded Room Comprising Discontinuous Layers Considering Permeability in Low Magnetic Field. IEEE Trans. Magn. 2014, 50, 1-4. [CrossRef]

7. Bork, J.; Hahlbohm, H.; Klein, R.; Schnabel, A. The 8-layered magnetically shielded room of the PTB: Design and construction. In Proceedings of the Biomag2000 Proceedings 12th International Conference on Biomagnetism, Helsinki, Finland, 13-17 August 2000; Publishing House: Espoo, Finland, 2001; pp. 970-973.

8. Altarev, I.; Babcock, E.; Beck, D.; Burghoff, M.; Chesnevskaya, S.; Chupp, T.; Degenkolb, S.; Fan, I.; Fierlinger, P.; Frei, A.; et al. A magnetically shielded room with ultra low residual field and gradient. Rev. Sci. Instrum. 2014, 85, 075106. [CrossRef] [PubMed]

9. Jiles, D.C.; Atherton, D.L. Theory of ferromagnetic hysteresis. J. Magn. Magn. Mater. 1986, 61, 48-60. [CrossRef]

10. Jiles, D.C.; Atherton, D.L. Theory of ferromagnetic hysteresis. J. Appl. Phys. 1984, 55, 2115-2120. [CrossRef]

11. Bergqvist, A. A simple vector generalization of the Jiles-Atherton model of hysteresis. IEEE Trans. Magn. 1996, 32, 4213-4215. [CrossRef]

12. Sun, Z.; Reisner, M.; Fierlinger, P.; Schnabel, A.; Stuiber, S.; Li, L. Dynamic modeling of the behavior of199permalloy for magnetic shielding. J. Appl. Phys. 2016, 199, 193902. [CrossRef]

13. Jiles, D.C. Frequency dependence of hysteresis curves in conducting magnetic materials. J. Appl. Phys. 1994, 76, 5849-5855. [CrossRef]

14. Cao, Q.; Pan, D.; Li, J.; Jin, Y.; Sun, Z.; Lin, S.; Yang, G.; Li, L. Optimization of a Coil System for Generating199Uniform Magnetic Fields inside a Cubic Magnetic Shield. Energies 2018, 11, 608. [CrossRef]

(C) 2019 by the authors. Licensee MDPI, Basel, Switzerland. This article is an open access article distributed under the terms and conditions of the Creative Commons Attribution (CC BY) license (http:/ / creativecommons.org/licenses/by/4.0/). 